\title{
Gamma Ray Photon Energy Absorption Buildup Factor Study In Some Soils
}

\author{
Jasbir Singh Dhillon ${ }^{1}$, Barjinderpal Singh ${ }^{2}$ and Gurdeep Singh Sidhu ${ }^{3}$ \\ ${ }_{1,2}^{2}$ Department of Physics, Singhania University, Rajasthan. (India) \\ ${ }^{3}$ Government Sports School, Ghudda (Bathinda) India.
}

\begin{abstract}
G.P. fitting method has been used to find energy absorption buildup factor for some soils taken from different states of India. Field of study has been spread over wide energy region 0.015-15.0 MeV up to a penetration depth of $40 \mathrm{mfp}$. Variation of EABF with incident photon energy and penetration depth has been studied. We observed that chosen soils have maximum value of EABF around $0.2 \mathrm{MeV}$. Variation in value of $E A B F$ was due to dominance of different interaction processes in different energy regions. A comparative study on the basis of different properties of selected soils like EBF, EABF, equivalent and effective atomic numbers has been also done.
\end{abstract}

Keywords- Energy absorption buildup factor (EABF), Exposure buildup factor (EBF), Mean free path (mfp), Shielding.

\section{Introduction}

Use of gamma rays in medicine, surgery, industry, research, agriculture etc. made our life full of excitement. At the same time nuclear accidents made human beings squeezed by dangerous diseases due to lack of proper knowledge of shielding materials. The study has been focused on buildup factor which plays a vital role during interaction of gamma rays with chosen soils. As gamma rays are very dangerous for human cells and other living organisms when exposed in excessive dose, selection of shielding materials becomes most important. Nuclear accidents due to natural and manmade disasters cannot be refused in future. People involved in industries, research laboratories and medical institutions may be victims of gamma ray exposure. For safety of these people and selection of shielding materials, buildup factor study becomes most important.

Also possibility of nuclear accidents in future in transportation of nuclear substances, storage of nuclear wastes, radiological terrorism etc. cannot be denied. So again we have to protect our self from gamma rays using safe and proper shielding materials in construction of houses. In the study of radiation dose received by the materials and dosimetry calculations, gamma ray buildup factor is of great importance. When gamma radiations interact with matter through Compton scattering, the energy of incident photon reduces and its direction also changes which results in creating scattered photons which can be estimated by the buildup factor. Buildup factor is a multiplicative factor used to obtain corrected response by including contribution of scattered photons to the Lambert-Beer's equation. There are two types of buildup factors naming energy absorption buildup factor (EABF) and exposure buildup factor (EBF). The energy absorption buildup factor is that in which the quantity of interest is the absorbed or deposited energy in the interacting material and the detector response function is that of absorption in the interacting material whereas the exposure buildup factor is that in which the quantity of interest is the exposure and the detector response function is that of absorption in air.

To calculate buildup factor there are different methods like G.P. fitting method, Harima et al. 1986 [1], invariant embedding method, Shimizu, 2002 [2]; Shimizu et al., 2004 [3], iterative method, Suteau and Chiron, 2005 [4] and Monte Carlo method, Sardari et al., 2009 [5]. American National Standards, ANSI/ANS 6.4.3., 1991 [6] calculated buildup factor for 23 elements, one compound and two mixtures viz. water, air and concrete at 25 standard energies in the energy range $0.015-15.0 \mathrm{MeV}$ up to penetration depth of 40 mean free path using G.P. fitting method. Hirayama and Tanaka,1985 [7] calculated the exposure buildup factor for plane isotropic, point isotropic and plane normal source by using PALLAS-PL, SP-Br code [8] in infinite and finite water shields in the energy range of $0.06 \mathrm{MeV}$ to $0.1 \mathrm{MeV}$. Harima et al. 1986 [1] also computed buildup factors using G.P. fitting method. Sakamoto et al., 1988 [9] compared the results of buildup factors for compounds with PALLAS code. Fujisawa 1994 [10] studied experimentally the buildup factor for multilayer of lead and aluminium for $0.5,1$ and $10 \mathrm{MeV}$ photon energies. Sidhu et al. 1998 [11] has attempted to generate buildup factor data for composite materials in the energy range of 0.015 -15.0 MeV. Shimizu et al. 2004 [3] compared the buildup factor values obtained by three different methods i.e. G.P. fitting, invariant embedding and Monte Carlo method for low-Z elements up to 100 mean free paths. D.Sardari and S. Baradaran 2010 [12] calculated buildup factor of gamma and X-ray photon the energy range of $0.2-2.0 \mathrm{MeV}$ in water and soft tissue using Monte Carlo code MCNP4C. The results are compared with buildup factor data of pure water. In each case very small deviation is observed. 
So, any of these methods/codes can be used to compute buildup factor data for low-Z materials. This data is of very great importance for engineers from the radiation shield designing point of view. In the present work buildup factor is computed using G.P. fitting method for some soils of different states of India. Energy region is selected from $0.015-15.0 \mathrm{MeV}$ up to a penetration depth of 40 mean free path $(\mathrm{mfp})$. The generated energy absorption buildup factor data has been studied as a function of incident photon energy and penetration depth. An attempt has been also made to perform a comparative study on the basis of different properties of selected soils like EBF, EABF, equivalent and effective atomic numbers.

\section{Selection of Materials}

\section{Materials and Methods}

For the present investigations five soil samples from different states of India are chosen and are given in TABLE1. The chemical composition of these soils is taken from world wide website of Krishiworld [13]. http://www.krishiworld.com/html/soil7.html

Soils are the basic raw materials used in construction of shelters, laboratories for nuclear experiments and residential houses. Soils can be used as good radiation shielding material because of their low cost and easy availability. As buildup factor data for these samples is not available in any form, so energy absorption and exposure buildup factor of the chosen soil samples has been calculated for incident photon energy from 0.015$15.0 \mathrm{MeV}$ and up to a penetration depth of $40 \mathrm{mfp}$.

Table1 Percentage Inorganic Composition of the Chosen Soil Samples.

\begin{tabular}{|c|c|c|c|c|c|}
\hline & Sandy & Loam & $\begin{array}{l}\text { Sandy } \\
\text { Loam }\end{array}$ & $\begin{array}{l}\text { Silt } \\
\text { Loam }\end{array}$ & Clay \\
\hline & Rajasthan & Uttar & Uttar & & Tamil \\
\hline & & Pradesh & Pradesh & Assam & $\mathrm{Nadu}$ \\
\hline Composition & S1 & S2 & S3 & S4 & S5 \\
\hline $\mathrm{SiO} 2$ & 91.72 & 86.98 & 84.84 & 86.49 & 65.16 \\
\hline $\mathrm{Al} 2 \mathrm{O} 3$ & 2.92 & 4.10 & 5.30 & 9.16 & 13.76 \\
\hline $\mathrm{Fe} 2 \mathrm{O} 3$ & 2.36 & 3.52 & 4.52 & 7.00 & 9.27 \\
\hline $\mathrm{MnO}$ & - & - & - & 0.21 & 0.25 \\
\hline $\mathrm{CaO}$ & 0.35 & 0.42 & 0.91 & 0.14 & 2.18 \\
\hline $\mathrm{MgO}$ & 0.78 & 1.29 & 0.52 & 0.90 & 2.47 \\
\hline $\mathrm{K} 2 \mathrm{O}$ & 0.33 & 0.56 & 0.16 & 1.85 & 0.14 \\
\hline $\mathrm{Na} 2 \mathrm{O}$ & 0.08 & - & 0.03 & - & 0.01 \\
\hline P2O5 & 0.08 & 0.06 & 0.10 & 0.24 & - \\
\hline
\end{tabular}

\section{Computational Work}

Buildup factors are computed using G.P. fitting parameters and the equivalent atomic number $Z_{\mathrm{eq}}$ of selected soils following three steps given below:

\subsection{Computation of equivalent atomic number $\left(Z_{\text {eq }}\right)$}

To compute equivalent atomic number of selected soils, the value of Compton partial attenuation coefficient $\left(\mu_{\text {compton }}\right)$ and total attenuation coefficient $\left(\mu_{\text {total }}\right)$ in $\mathrm{cm}^{2} / \mathrm{g}$ are obtained for selected soils in the energy range of 0.015 to $15.0 \mathrm{MeV}$ and also for elements from $\mathrm{Z}=1$ to $\mathrm{Z}=40$ in the same energy range by using the state of art and convenient computer program WinXCOM computer program Gerward et al. 2001 [14]; Gerward et al. 2004 [15]) initially developed as XCOM, Berger and Hubbel, 1999 [16]. Ratio R ( $\mu$ compton / $\mu$ total) for selected soil samples and for elements from $Z=1$ to 40 is calculated at energies 0.015 to $15.0 \mathrm{MeV}$ using a simple 
Gamma Ray Photon Energy Absorption Buildup Factor Study In Some Soils

computer program. For the interpolation of $\mathrm{Z}_{\mathrm{eq}}$ for selected samples, the ratio $\mathrm{R}$ of particular sample at a given energy is matched with the corresponding ratio of elements at the same energy. For the case, where the ratio $R$ lies between two successive ratios of known elements, the value of $Z_{\mathrm{eq}}$ is interpolated using following formula, Sidhu et al., 2000 [17]

$$
Z_{\mathrm{eq}}=\frac{Z_{1}\left(\log R_{2}-\log R\right)+Z_{2}\left(\log R-\log R_{1}\right)}{\log R_{2}-\log R_{1}}
$$

where $Z_{1}$ and $Z_{2}$ are the atomic numbers of elements corresponding to ratio $R_{1}$ and $R_{2}$. The computed values of $Z_{\text {eq }}$ for different soils samples are given in Table 2 .

Table2. Equivalent Atomic Number $\left(Z_{\mathrm{eq}}\right)$ of Chosen Soil Samples in Energy Range of $0.015-15.0 \mathrm{MeV}$

\begin{tabular}{|c|c|c|c|c|c|}
\hline \multirow[t]{2}{*}{$\mathrm{E}(\mathrm{MeV})$} & \multicolumn{5}{|c|}{ Equivalent atomic number $\left(Z_{\mathrm{eq}}\right)$} \\
\hline & S1 & S2 & $\mathbf{S 3}$ & S4 & S5 \\
\hline 0.015 & 12.09 & 12.34 & 12.57 & 12.96 & 13.54 \\
\hline 0.02 & 12.16 & 12.43 & 12.68 & 13.09 & 13.7 \\
\hline 0.03 & 12.27 & 12.57 & 12.79 & 13.26 & 13.9 \\
\hline 0.04 & 12.33 & 12.65 & 12.88 & 13.35 & 14.02 \\
\hline 0.05 & 12.4 & 12.71 & 12.97 & 13.47 & 14.1 \\
\hline 0.06 & 12.43 & 12.73 & 12.99 & 13.53 & 14.2 \\
\hline 0.08 & 12.45 & 12.75 & 13.03 & 13.6 & 14.21 \\
\hline 0.1 & 12.63 & 13.05 & 13.2 & 13.66 & 14.44 \\
\hline 0.15 & 12.85 & 12.85 & 13.44 & 13.44 & 13.98 \\
\hline 0.2 & 12.92 & 12.92 & 12.92 & 12.92 & 14.49 \\
\hline 0.3 & 12.5 & 12.5 & 12.5 & 12.5 & 14.5 \\
\hline 0.4 & 12.5 & 12.5 & 12.5 & 12.5 & 14.5 \\
\hline 0.5 & 12.5 & 12.5 & 12.5 & 12.5 & 14.5 \\
\hline 0.6 & 12.5 & 12.5 & 12.5 & 12.5 & 14.5 \\
\hline 0.8 & 12.5 & 12.5 & 12.5 & 12.5 & 14.5 \\
\hline 1 & 12.5 & 12.5 & 12.5 & 12.5 & 14.5 \\
\hline 1.5 & 12.5 & 12.5 & 12.5 & 12.5 & 14.5 \\
\hline 2 & 9.726 & 9.726 & 9.741 & 12.87 & 12.88 \\
\hline 3 & 10.69 & 10.69 & 10.69 & 11.59 & 11.72 \\
\hline 4 & 10.93 & 11.41 & 11.41 & 11.41 & 11.94 \\
\hline 5 & 11.17 & 11.17 & 11.17 & 11.61 & 11.99 \\
\hline 6 & 11.27 & 11.27 & 11.27 & 11.47 & 11.72 \\
\hline 8 & 11.18 & 11.18 & 11.44 & 11.44 & 12.02 \\
\hline 10 & 10.79 & 11.18 & 11.36 & 11.55 & 11.73 \\
\hline 15 & 10.99 & 11.12 & 11.36 & 11.63 & 11.89 \\
\hline
\end{tabular}

\subsection{Computation of G.P. fitting Parameters}

American National Standard 1991, ANSI/ANS- 6.4.3 [6] has provided the exposure and energy absorption G.P. fitting parameters of twenty three elements $(\mathrm{Ca}, \mathrm{Fe}, \mathrm{Si}$ etc.) in the energy range of $0.015-15.0$ $\mathrm{MeV}$ and up to $40 \mathrm{mfp}$. The computed values of $Z_{\mathrm{eq}}$ for selected soils were used to interpolate G.P. fitting parameters $\left(\mathrm{b}, \mathrm{c}, \mathrm{a}, \mathrm{X}_{\mathrm{k}} \mathrm{d}\right)$ for the exposure and energy absorption buildup factor in the chosen energy range of 0.015-15.0 MeV and penetration depth (1-40 mfp). The formula, Sidhu et al., 2000 [17] used for the purpose of interpolation of the G.P. fitting parameters is given below:

$$
P=\frac{P_{1}\left(\log Z_{2}-\log Z_{e q}\right)+P_{2}\left(\log Z_{e q}-\log Z_{1}\right)}{\log Z_{2}-\log Z_{1}}
$$

where $P_{1}$ and $P_{2}$ are the values of G.P. fitting parameters corresponding to atomic number $Z_{1}$ and $Z_{2}$ respectively at a given energy and $Z_{\text {eq }}$ is the equivalent atomic number of chosen soils at same energy. $Z_{1}, Z_{2}$ are the successive elemental atomic numbers such that

\subsection{Computation of buildup factor}

$$
Z_{1}<Z_{e q}<Z_{2}
$$

In this last step computed G.P. fitting parameters of selected soils are used to calculate buildup factors. Following formulae, Harima et al., 1986 [1] are used. 


$$
\begin{gathered}
\mathrm{B}(\mathrm{E}, \mathrm{x})=1+\frac{(\mathrm{b}-1)\left(\mathrm{K}^{\mathrm{x}}-1\right)}{\mathrm{K}-1} \quad \text { for } \mathrm{K} \neq 1 \\
\mathrm{~B}(\mathrm{E}, \mathrm{x})=1+(\mathrm{b}-1) \mathrm{x} \quad \text { for } \mathrm{K}=1 \\
\mathrm{~K}(\mathrm{E}, \mathrm{x})=\mathrm{cx}{ }^{\mathrm{a}}+\mathrm{d} \frac{\tanh \left(\mathrm{x} / \mathrm{X}_{\mathrm{k}}-2\right)-\tanh (-2)}{1-\tanh (-2)} \quad \mathrm{x} \leq 40 \mathrm{mfp}
\end{gathered}
$$

where $\mathrm{a}, \mathrm{b}, \mathrm{c}, \mathrm{d}$, and $\mathrm{X}_{\mathrm{K}}$ are the G.P. fitting parameters that depend upon attenuating medium and source energy, $x$ is the distance between source and detector in the medium. $E$ is the incident photon energy. $b$ is buildup factor at $1 \mathrm{mfp}$. $\mathrm{K}$ is the photon dose multiplication and change in the shape of spectrum with increasing penetration depth. $\mathrm{K}$ is represented by tangent hyperbolic function of penetration depth in $\mathrm{mfp}$.

To standardize this interpolation method, exposure and energy absorption buildup factor for water are computed up to $40 \mathrm{mfp}$ in energy range of $0.015-15 \mathrm{MeV}$ with this method. The results so obtained are compared with the result of ANSI/ANS 6.4.3 standard, Sidhu et al. 2000 [17] for a few randomly selected energies of $0.015,5.0,10.0,15.0 \mathrm{MeV}$. The compared values of EBF and EABF of water have been plotted and shown in figure 1. The two results are in good agreement within the limits of statistical error. Thus we can assume safely that the present method is appropriate and suitable for calculation of exposure and energy absorption buildup factor of chosen soils.

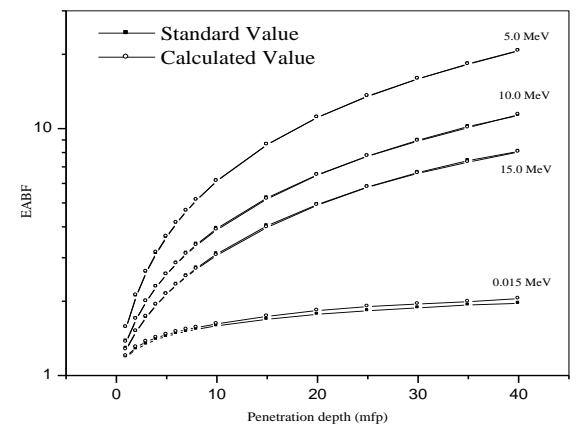

Fig. 1(a)

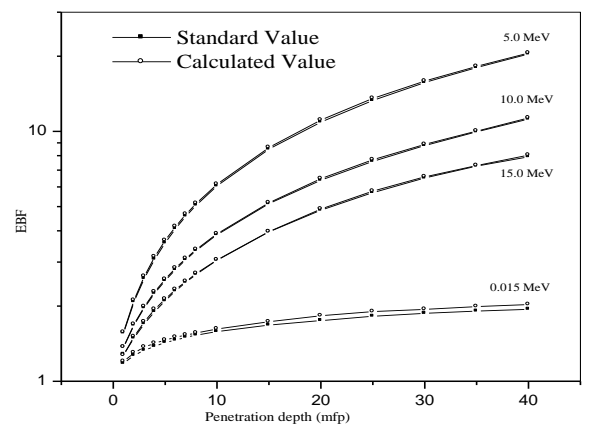

Fig. 1(b)

Fig. 1(a,b) Standardization of the G-P fitting method by comparison of calculated and standard values of EABF and $\mathrm{EBF}$ for water.

\section{Results And Discussions}

The energy and penetration depth dependence of energy absorption buildup factor and comparison study of EBF and EABF have been discussed in following sub sections.

\section{Effect of Incident Photon Energy on Energy Absorption Buildup Factor}

All the soil samples show almost similar behavior at different mean free paths. Energy absorption buildup factor (EABF) is comparatively smaller for incident photon energy less than $\mathrm{E}_{\mathrm{pe}}$ for all soil samples at different penetration depths. The reason behind this is that at lower incident photon energies, photoelectric absorption takes place and photons are completely absorbed by the material Here $\mathrm{E}_{\mathrm{pe}}$ is the incident photon energy at which the photoelectric interaction coefficient matches the Compton interaction coefficient for a given soil sample. It is observed that for incident photon energy $\mathrm{E}_{\mathrm{pe}}<\mathrm{E}<\mathrm{E}_{\mathrm{pp}}$ Compton scattering starts dominating photoelectric absorption. Here $\mathrm{E}_{\mathrm{pp}}$ is the incident photon energy value at which the pair production interaction coefficient matches the Compton interaction coefficient for a particular soil sample. At higher energies pair production dominates Compton scattering resulting in lowering of buildup factor. Fig.2 describes this graphically. The approximate values of $\mathrm{E}_{\mathrm{pe}}, \mathrm{E}_{\mathrm{pp}}$ and $\mathrm{E}_{\text {peak }}$ are given in TABLE 3.

Table3.Values of $E_{p e}, E_{p p}$, and $E_{p e a k}$ For Selected Soil Samples.

\begin{tabular}{|l|l|l|l|}
\hline Soil Sample & $\begin{array}{l}\mathbf{E}_{\mathbf{p e}} \\
(\mathbf{M e V})\end{array}$ & $\begin{array}{l}\mathbf{E}_{\mathbf{p p}} \\
(\mathbf{M e V})\end{array}$ & $\begin{array}{l}\mathbf{E}_{\text {peak }} \\
(\mathbf{M e V})\end{array}$ \\
\hline Sandy Rajasthan (S1) & 0.15 & 1.5 & 0.20 \\
\hline Loam Uttar Pradesh (S2) & 0.15 & 1.5 & 0.20 \\
\hline Sandy Loam Uttar Pradesh (S3) & 0.15 & 1.5 & 0.20 \\
\hline Silt Loam Assam (S4) & 0.20 & 1.5 & 0.20 \\
\hline Clay Tamil Nadu (S5) & 0.20 & 1.5 & 0.30 \\
\hline
\end{tabular}




\section{Effect of Penetration Depth on Energy Absorption Buildup Factor}

The curves at different energies in figure 3 show that there is continuous increase in EABF with increase in penetration depth for all samples. It is due to the fact that the increase in penetration depth increases the interaction of gamma-radiation photons with matter resulting in generation of large number of low energy photons due to occurrence of Compton scattering process.

It is also noted from the figure that for a fixed value of penetration depth, the buildup factor increases with increase in incident photon energy from 0.015 to $0.3 \mathrm{MeV}$. The buildup factor values are highest at 0.3 $\mathrm{MeV}$ after which the buildup factor decreases with the increase in incident photon energies from 0.6 to 15.0 $\mathrm{MeV}$. It is seen that for energies greater than $1.0 \mathrm{MeV}$, there is a sharp fall in the value of buildup factor which ultimately depicts the dominance of pair production process in the energy region.
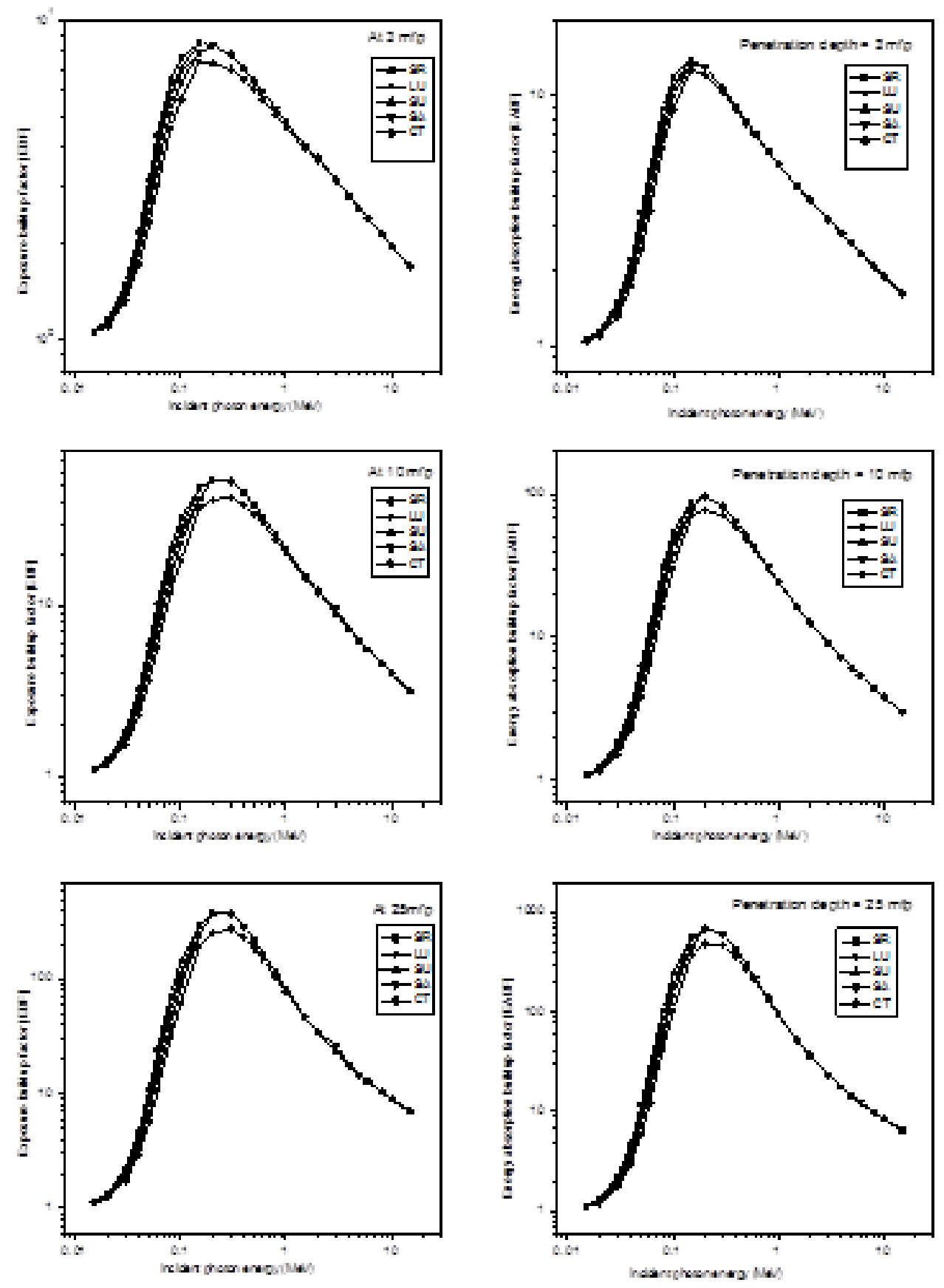

Fig2. The EABF and EBF values for all samples in the energy region $0.015-15.0 \mathrm{MeV}$ at $3 \mathrm{mfp}, 10 \mathrm{mfp}$ and 25 mfp. 

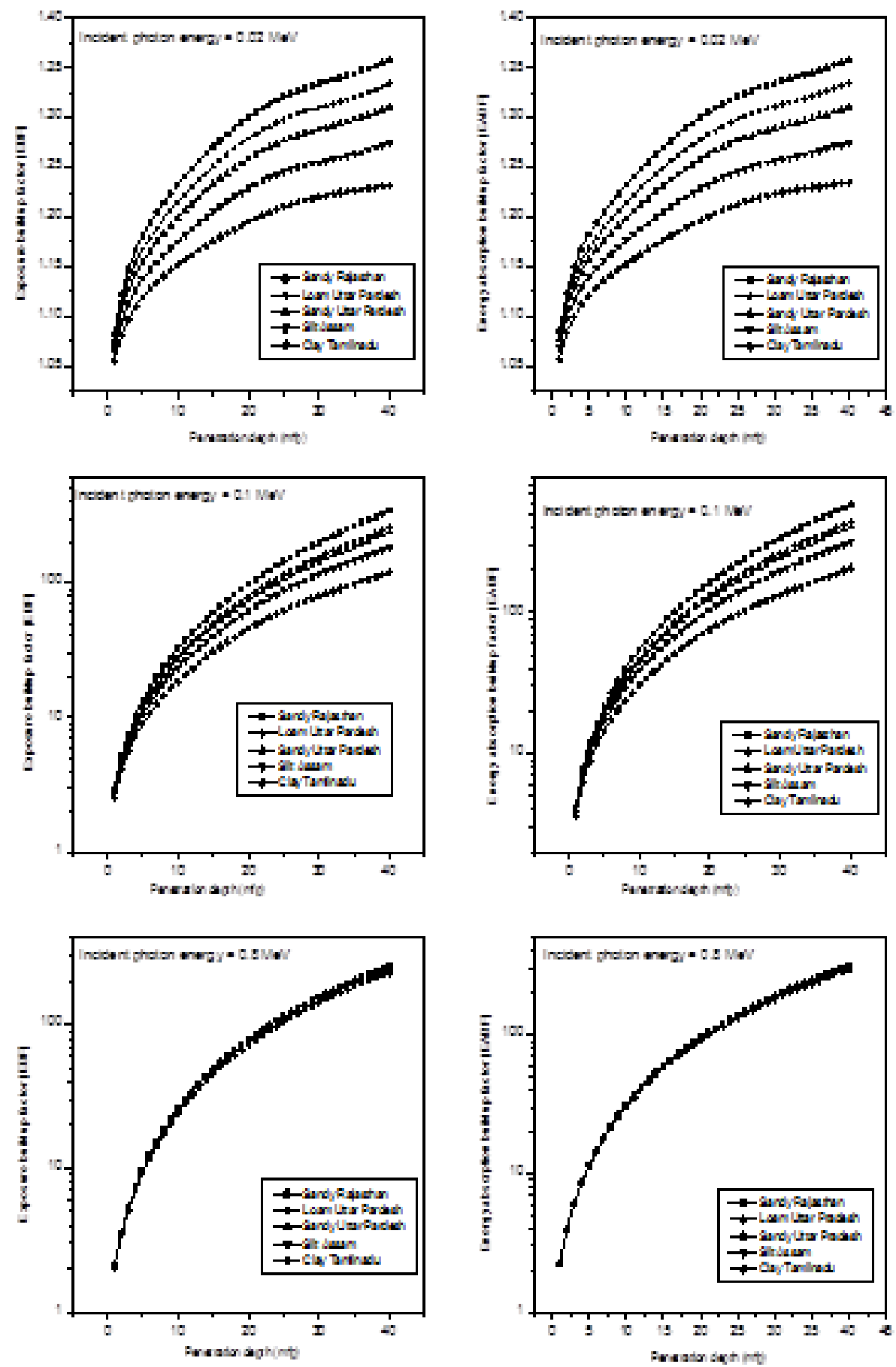

Fig.3. The EABF and EBF values for all samples up to penetration depth of $40 \mathrm{mfp}$ at $0.02 \mathrm{MeV}, 0.1 \mathrm{MeV}$ and $0.8 \mathrm{MeV}$.

\section{Dependence of EABF on $Z_{\text {eff }}$}

To study the average behavior of a sample, effective atomic number $Z_{\text {eff }}$ of the sample is obtained as an average of equivalent numbers over incident photon energies. From graphs shown in Fig.4, it is found that for energy region (0.3-15.0 MeV) the values of buildup factors show a decreasing trend with increase in the value of $Z_{\text {eff }}$. It is evident that for energy greater than $1.0 \mathrm{MeV}$ there is practically no change in values of EABF. Thus at higher energies $E A B F$ are independent of $Z_{\text {eff. }}$ Also with increase in penetration depth, probability of multiple scattering increases and creating more photons increases the value of EABF. 

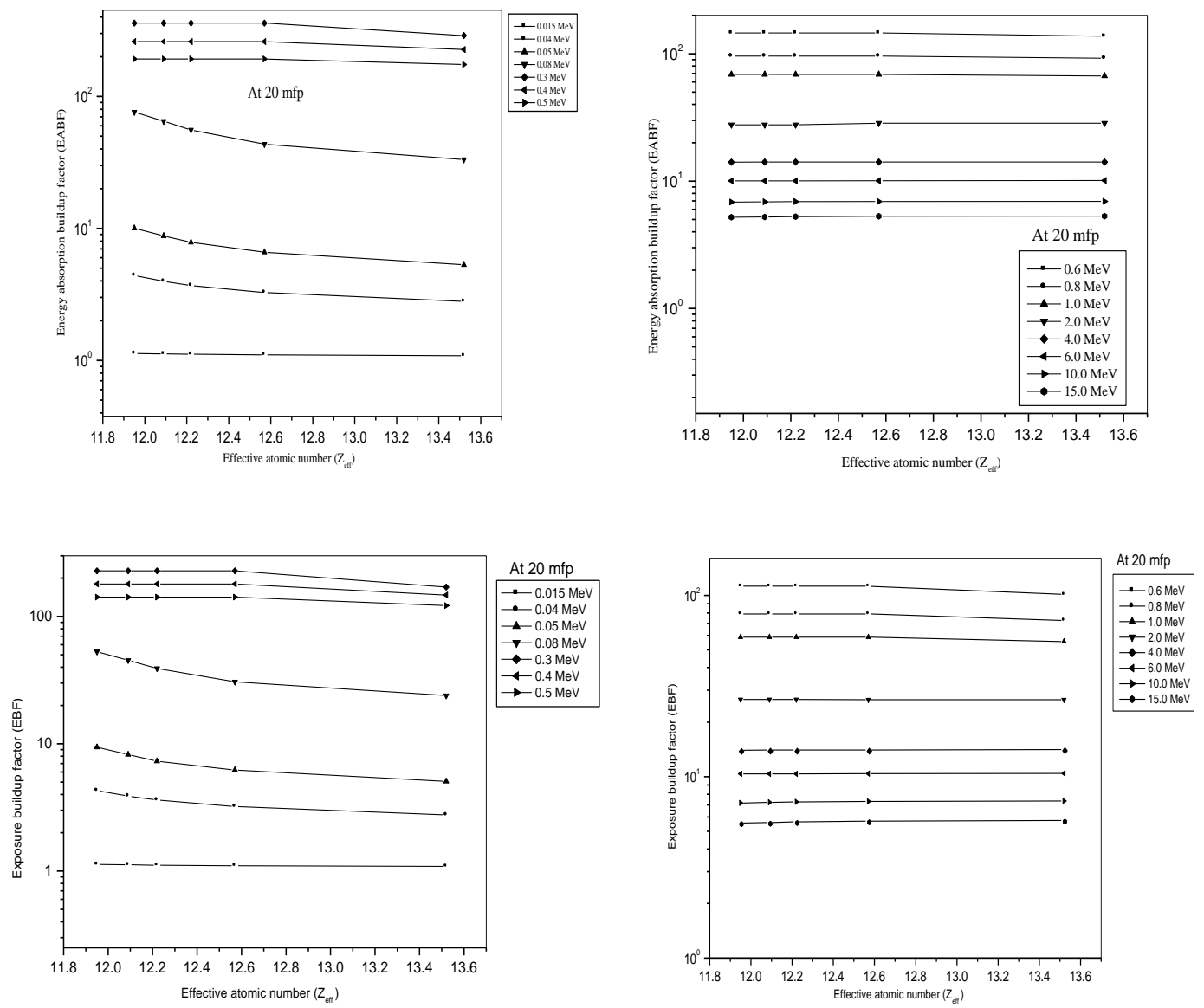

Fig. 4 (a,b,c,d)The variation of EABF and EBF of chosen soils with effective atomic numbers for energies 0.015- $15.0 \mathrm{MeV}$ at $20 \mathrm{mfp}$.

\section{Comparison of EABF and $E B F$}

Observations concluded that the values of EABF and EBF show similar behavior towards incident photon energy, penetration depth and some other properties of chosen soils. The significant differences observed are between the values of EABF and EBF at some photon energies for different samples. These differences estimate the location of maximum radiation occurrence. The maximum differences in the values confine to intermediate energy region due to dominance of Compton scattering over other interaction processes. It is observed that the values of $\mathrm{EABF}$ are significantly larger than $\mathrm{EBF}$ for materials having high equivalent atomic number whereas materials with low equivalent atomic number have larger EBF than EABF. This indicates that for materials of high $\mathrm{Z}_{\mathrm{eq}}$, the absorption inside the medium is much more as compared to air. The absorption in air contributes more to the EBF than EABF for materials of low $\mathrm{Z}_{\mathrm{eq}}$.

\section{Conclusions}

This study of buildup factor of soils will be helpful in estimating the transport and degradation of gamma radiations in these soils. Mostly Lead and Mercury are used as shielding materials. But these are difficult to use at large scale due to their higher cost and availability. Soils can be used as a gamma-ray shielding material in field experiments which is suitable from the point of view of cost and availability. Above studies projects Soil as a potential radiation shielding material.

\section{Acknowledgment}

We are grateful to Berger and Hubbell for providing the convenient computer program XCOM with the help of National Institute of Standards and Technology, Gaithersburg. 


\section{References}

[1]. Harima, Y., Sakamoto, Y, et al., 1986. Validity of the geometric progression formula in approximating the gamma ray buildup factors Nucl. Sci.Eng. 94, 24 -35.

[2]. Shimizu, A., 2002. Calculations of gamma ray buildup factors up to depths of $100 \mathrm{mfp}$ by the method of invariant embedding, (I) analysis of accuracy and comparison with other data. J.Nucl Sci. Technol. 39, 477- 486.

[3]. Shimizu, A., Onda, T., Sakamoto, Y., 2004. Calculations of gamma ray buildup factors up to depths of $100 \mathrm{mfp}$ by the method of invariant embedding, (III) generation of an improved data set. J. Nucl. Sci. Technol. 41, 413 - 424.

[4]. Suteau, C., Chiron, M., 2005. An iterative method for calculating gamma ray buildup factors in multi-layer shields. Radiat. Prot. Dosim. 116, $489-492$.

[5]. Sardari,D., Abbaspour,A., Baradaran, S., Babapour, F., 2009. Estimation of gamma and X-ray photons buildup factor in soft tissue with Monte Carlo method. Appl. Radiat. Isot. 67, 1438 - 1440.

[6]. ANSI,1991. American National Standard Gamma-Ray Attenuation Coefficient and Buildup Factors for Engineering Materials. ANSI/ANS-6.4.3.

[7]. Harima, Y. and Tanaka, S. (1985) A study of buildup factors, angular and energy distribution at small distances from three source geometries- plane isotropic, point isotropic and plane normal for low energy gamma rays incident on water. Nucl. Sci. Engg. 90,165.

[8]. Takeuchi, K. and Tanaka, S. PALLAS-ID (VII). A Code for direct integration of transport equation in one-dimensional plane and spherical geometries. JAERI-M 84, 214 (1984).

[9]. Sakamoto, Y., Tanaka, S., Harima, Y., 1988. Interpolation of gamma ray build-up factors for point isotropic source with respect to atomic number. Nucl. Sci. Eng. 100, 33 - 42.

[10]. Fujisawa,K (1994) Parametric study of shielding codes used for Packaging Ramtrans 5,215 G. S. Sidhu, Parjit S. Singh and Gurmel Singh Mudahar and G.S. Brar and Makhan singh, 1998. An interpolation method to generate buildup factor data of composite materials. NSRP (National symposium on radiation physics) 12.

[11]. Sardari,D and S.baradaran (2010) Semi empirical relationship for photon buildup factor in soft tissue and water. Radiation protection dosimetry 10.1093/rpd/ncq 212 http://www.krishiworld.com/html/soil7.html Chemical composition of Indian soils.

[12]. Gerward, L., Guilbert, N., Jensen, K. B., Levring, H., 2001. X-ray absorption in matter.

[13]. Reengineering XCOM. Radiat. Phys. Chem. 60, 23-24.

[14]. Gerward, L., Guilbert, N., Jensen, K. B., Levring, H. et al., 2004. Win XCom- A program for calculating attenuation coeffiecients. Radiat. Phys. Chem. 71, 653-654

[15]. Berger, M.J., Hubbell, J.H., 1987/1999. XCOM: Photon Cross-Sections Database, Web Version 1.2, National Institute of Standards and Technology, Gaithersburg, MD20899, USA. http://physics.nist.gov/xcom (Originally published as NBSIR 87-3597 "XCOM: Photon Cross Sections on a Personal Computer")

[16]. G. S.Sidhu, Karamjeet Singh, Parjit S. Singh and Gurmel S. Mudahar. (2000). Effect of absorber thickness and beam divergence on gamma-ray buildup factor. Indian J. Phys. 74A (5), 505- 507. 